\title{
The effects of constrained rehearsal on judgments of temporal order
}

\author{
BRYAN C. AUDAY, CHRISTOPHER SULLIVAN, and HENRY A. CROSS \\ Colorado State University, Fort Collins, Colorado
}

\begin{abstract}
Tzeng and Cotton's (1980) study-phase retrieval model holds that temporal encoding of words is acquired through the establishment of contextual associations among list items. Such associations are strengthened by a subject's ability to rehearse previous items, that is, to engage in displaced rehearsals. Since displaced rehearsals are critical in the study-phase model, to place constraints on such rehearsals should alter performance for the memory of temporal order. Five different rehearsal strategies were employed within a distractor paradigm to test this prediction. Two distinct instructional sets were also administered, with one half of the subjects instructed that serial order information was important, while the remaining subjects were told only that a free recall test would be given. Differences were found for serial position scores among the different rehearsal strategies in support of the study-phase retrieval model. Informed and uninformed instructional groups did not differ in temporal memory.
\end{abstract}

To understand the mechanism or mechanisms responsible for the encoding and retrieval of temporal order information is an important step in the elucidation of memory processes (Tulving \& Madigan, 1970; Tzeng, Lee, \& Wetzel, 1979). In the literature, the study of temporal order has comprised three areas: (1) the coding of an item's position within a list (Zimmerman \& Underwood, 1968), (2) the establishment of order relationships among list items (Yntema \& Trask, 1963), and (3) time duration (Block, 1982; Fraisse, 1984). The primary focus of these investigations has involved both the establishment of order relationships among words and judgments of recency.

Yntema and Trask (1963) asked subjects to discriminate the most recent item (i.e., to make a judgment of recency) in a word pair after the presentation of a long list. They proposed a time-tag theory, according to which stimuli, as they become encoded, are assigned separate time tags. Judgments of recency are thus made because each item carries temporal information.

Hinrichs (1970) proposed a trace strength theory for judgments of recency, according to which subjects do not store temporal information as they encode; instead, encoded items acquire traces that decay unidimensionally in strength over time. Not only is decay important to this theory, but decision is also significant. Hinrichs applied the theory to judgments of recency after acknowledging its usefulness in explaining other decision models of memory (Kintsch, 1967). Hinrichs's theory has been criticized (Hasher \& Zacks, 1979; Hintzman, Block, \& Summers, 1973; Toglia \& Kimble, 1976; Tulving \& Madigan, 1970), in part for its inability to account for the well-documented primacy effect (Tzeng \& Cotton, 1980).

B. C. Auday's present address is Department of Psychology, Gordon College, Grapevine Rd., Wenham, MA 01984.
Murdock's (1974) conveyor-belt model of memory has also been invoked to explain judgments of recency. All memories are based on a temporal component, whereby items placed on the conveyor belt become less distinctive as they are gradually moved away. Murdock's theory too cannot account for the primacy effect or explain how displaced or subjective rehearsals fail to interrupt the temporal relationships (Tzeng \& Cotton, 1980).

Tzeng (1976), deriving his explanation from Yntema and Trask's (1963) time-tag concept, proposed that an item receives a time tag upon entering the informationprocessing system. Such a tag enjoys "precedence" and is immune to later interfering effects, including displaced rehearsals. Subsequently, Tzeng, Lee, and Wetzel (1979), and Tzeng and Cotton (1980), proposed the study-phase retrieval model. Basically, this model has two components. First, the temporal code is established. During acquisition, "the temporal relationship between $A$ and $B$ is established only when $A$ is available at the time when $B$ is encoded. Since $B$ is a current item, $A$ must be a prior item by definition"' (Tzeng \& Cotton, 1980, p. 714). The second component refers to the study-phase retrieval mechanism. As items enter the memory set, the subject automatically rehearses previous items. These displaced rehearsals, which comprise the study-phase mechanism, are critical for the establishment of order relationships among intralist items. This model allows items to become contextually associated with the "cognitive context (i.e., previously presented items; the subject's mood, state, or attitude; some conspicuous external stimuli, etc.) of the learning environment' (Tzeng \& Cotton, 1980, p. 706). Displaced rehearsals strengthen the contextual associations among items, which enhances memory for serial order. Whereas displaced rehearsals become a stumbling block for the trace-strength, conveyor-belt, and precedence theories, they play an important role in the study-phase retrieval theory. 
Memory processes vary in the attentional resources required (Schneider \& Shiffrin, 1977; Shiffrin \& Schneider, 1977). In general, temporal order information has been thought to be encoded automatically (Hasher \& Zacks, 1979; Toglia \& Kimble, 1976; Tzeng \& Cotton, 1980; Zimmerman \& Underwood, 1968). However, a growing body of research has raised questions about the automaticity of many memory processes (Greene, 1984; NavehBenjamin, 1987; Naveh-Benjamin \& Jonides, 1986; Sanders, Gonzalez, Murphy, Liddle, \& Vitina, 1987; Williams \& Durso, 1986), thus forcing a reexamination of the intentionality criteria of Hasher and Zacks. For example, Michon and Jackson (1984) have found differences between a group receiving recognition instructions and another group given temporal order instructions.

The present investigation tested the theory that normal displaced rehearsals are critical in the establishment of temporal order in a procedure that has disrupted such rehearsals. In a personal communication (April 1985), Tzeng has acknowledged that manipulating a subject's ability to engage in displaced rehearsals should influence performance in a test of order information. The encoding of temporal order should be hampered as constraints are placed upon the subject's ability to use effective displaced rehearsals. Additionally, the study-phase model predicts that "knowledge about the recency-judgment task beforehand would not improve the judgment performance" (Tzeng \& Cotton, 1980, p. 714). More current research, however, leads to the prediction that subjects informed that serial order information will be tested will perform better than subjects not so informed (Michon \& Jackson, 1984).

\section{METHOD}

\section{Subjects}

One hundred and sixty introductory psychology students from Colorado State University participated in the study and received credit to fulfill a course requirement. There were 92 females and 68 males.

\section{Materials}

Words. Ninety-six words of AA frequency were chosen from Thorndike and Lorge (1944). The words were from four to seven letters long, were relatively homogeneous (4.33-6.73) in terms of imagery (Paivio, Yuille, \& Madigan, 1968), and were minimally related associatively (Palermo \& Jenkins, 1964). Forty-eight words were randomly selected to make up the test list, and two computer-generated randomizations of this list were constructed. The remaining $\mathbf{4 8}$ words constituted a nontest list which was used in a rehearsal situation.

Free recall test. Each subject was required to write as many words as he or she could remember from the test list without concern for order.

Serial position test. Forty-eight words were randomly presented on a test page in three columns. Instructions at the top of the page stated that the test list had been divided into eight "blocks" with each block encompassing six serial positions. For example, Block 1 represented the first 6 words in the list. Next to each word, subjects recorded the block in which they believed that word had occurred.

Judgment of recency test. The $\mathbf{4 8}$ words in the test list were grouped into 24 pairs of words and placed on a separate page. The word pairs were formed on the basis of two criteria. First, each word pair had to have either 0,2 , or 4 intervening test items between them. For example, if the 16th test word made up one half of the word pair, it could be combined with the 15 th or 17 th test word ( 0 intervening items), or the 13th or 19th word ( 2 intervening items), or the 11 th or 21 st word
(4 intervening items). Second, all word pairs had to be contained within one of the following four serial locations: (1) Positions 1-12, (2) Positions 13-24, (3) Positions 25-36, (4) Positions 37-48. Thus, a word could not be paired with words that were members of a distant location. The judgment of recency test was constructed in this way in order to test whether the variables were operating consistently throughout the list. The subjects were instructed to circle the word from each pair that was more recent (i.e., that occurred farther along in the list). The correct word occurred on the right or left an equal number of times, and the word pairs were randomly distributed on the page.

\section{Apparatus}

The experiment was held in a large laboratory $(6.1 \times 5.79 \mathrm{~m})$ that accommodated four test stations with Apple microcomputers. Each distinct treatment used all the monitors an equal number of times. In addition, four Sony reel-to-reel tape recorders were used (model no. TC-106A, distributed by Superscope, Los Angeles, California) to record the subjects' rehearsals.

\section{Experimental Design and Procedure}

The design for the experiment was a 2 (informed vs. uninformed instructions) $\times 5$ (five rehearsal conditions) $\times 2$ (two different orders for the memory tests) between-subjects factorial. Eight subjects served in each of the 20 basic conditions. Subgroups of two, three, or four subjects were run at a time, with each subject at his or her own test station. As each subgroup entered the laboratory, the group was randomly assigned to one of the conditions. Care was taken to isolate the subjects from one another by placing partitions between adjacent test stations.

The experimenter read the appropriate subgroup instructions, and the subjects were informed that $\mathbf{4 8}$ words would be presented, one at a time, on the computer screen and that each test word would remain on the screen for $2 \mathrm{sec}$. After each word's appearance, an interstimulus interval (ISI) of $5.5 \mathrm{sec}$ occurred before the next word appeared. One half of the subjects were instructed to remember as many of the $\mathbf{4 8}$ words as possible for a subsequent free recall test, and to remember the serial order of each word that occurred within the list (informed group). The remaining subjects were instructed that they would be required to recall as many words as they could remember, but there was no reference to a serial order or a judgment of recency (JOR) test (uninformed group). Each word was displayed inside a $5.5 \mathrm{~cm}$ wide and $2.5 \mathrm{~cm}$ high stimulus presentation box that was centered on the screen. Below the box, two $4 \mathrm{~cm}$ lines were displayed. A brief practice trial using two stimulus words was given.

Each subject was asked to engage in one of five different rehearsal strategies during the various ISIs. This manipulation constituted the five rehearsal groups as follows: Free Rehearsal (FR), Distractor Rehearsal (DR), Forward Rehearsal (FWR), Backward Rehearsal (BWR), Subjective Rehearsal (SR). FR subjects were given the 48 test words, one at a time, and were instructed to rehearse audibly any previous words they could remember, two at a time, during the ISIs. FR subjects were encouraged to visualize the 2 rehearsal words as appearing on the lines below the stimulus presentation box.

Subjects in the DR group were induced to rehearse word pairs that were not members of the test list. These words appeared for $1.1 \mathrm{sec}$ just above the blank lines during the ISI. During a single ISI, a given word pair was presented twice, and these same word pairs occurred over four consecutive ISIs. A total of $\mathbf{2 4}$ different pairs of distractor words were used.

The FWR group was similar to the DR group, except that the FWR subjects were required to rehearse test items in a forward serial pattern. For example, during the initial ISI, the 1st word pair to be rehearsed was made up of the 1 st and 2 nd test words. This word pair was repeated twice in a given ISI and continued for four consecutive ISIs. The 1st word pair was then followed by the 2 nd word pair, which was composed of the 3rd and 4th test words, and this forward serial pattern was maintained throughout the test list.

The BWR group was like the FWR group except that the 1st word pair was comprised of the 48th and 47th test words, respectively, and the 2 nd word pair included the 46 th and 45 th words, respectively. The rehearsal words initially came from the end of the test list and worked their way successively back through the list to the beginning. 
The SR group was used as a control. These subjects were not constrained to rehearse test or nontest words and were free to use the ISI in any way they chose. In all rehearsal groups except the SR group, the subjects rehearsed the word pairs into a microphone.

After the list was presented, all the subjects were administered a free recall test. Subsequently, the subjects received either a serial position test followed by a judgment of recency test (Order 1), or a judgment of recency test followed by a serial position test (Order 2).

\section{RESULTS}

\section{Free-Recall Scores}

A $2 \times 5$ between-subjects analysis of variance was conducted on the mean number of words free recalled from the test list, with the two instructional groups tested across five different rehearsal conditions. Since free recall scores were obtained first, we were able to combine the groups that received the two test orders mentioned earlier.

The main effect for instructional group was significant, $[F(1,150)=4.11, p<.05]$, with subjects in the uninformed group recalling more words $(M=15.25$ vs. $M$ $=13.51)$. A significant main effect for rehearsal groups was found $[F(4,150)=22.66, p<.001]$, and a Newman-Keuls post hoc test revealed that the SR group recalled more words than did all other conditions. Conversely, the DR group recalled fewer words than did all the other groups. There were no other mean differences, and no interaction was found for instruction condition $x$ rehearsal group.

\section{Serial Position Scores}

A $2 \times 5 \times 2$ between-subjects analysis of variance was performed on Pearson $r$-correlation coefficients. Each coefficient was obtained by correlating the subject's judged block (Blocks 1-8) with the actual block in which the item had occurred. Each subject contributed one coefficient that became an index of how accurately he or she rated the serial position of the test words.

The analysis did not reveal a difference for the instruction variable. Differences were found among the five different rehearsal groups $[F(4,140)=18.68, p<.001]$. The group means for the FR, DR, FWR, BWR, and SR rehearsal groups were $.360, .278, .327, .079$, and .363 , respectively. The main effect due to test order was not significant. The first-order interaction of instruction $\times$ rehearsal group was not significant, nor was the instruction $\times$ test order interaction. However, the interaction of rehearsal group $\times$ test order was significant $[F(4,140)$ $=2.95, p<.01]$. Newman-Keuls post hoc analysis revealed that the mean scores for the BWR condition when administered the two test orders were significantly lower than all other conditions. No other mean comparisons were significant, and the second-order interaction of instruction $\times$ rehearsal group $\times$ test order was not significant.

\section{Judgment of Recency Test}

Total JOR scores. A $2 \times 5$ between-subjects analysis was conducted on the total number of correct JOR scores (it was possible for subjects to contribute a low score of
0 and a maximum score of 24). Test order was eliminated from the analysis because the main effect of test order was not significant, nor did it interact with any other variable.

As in the results obtained from the serial position dependent variable, the analysis of variance did not reveal a main effect for the instruction variable using JOR scores. The means for the informed and uninformed groups were 13.18 and 12.64 , respectively. Unexpectedly, the comparison among the five different rehearsal conditions showed no differences in JOR scores. The means for the FR, DR, FWR, BWR, and SR groups were 13.00, 12.26, $13.38,12.53$, and 13.38 , respectively. No significant interaction was found for instruction $\times$ rehearsal group.

Semi-total JOR scores. It was possible to determine whether the independent variables had a different influence on words in the beginning of the test list (primacy effect, Positions 1-12) than on words at the end of the list (recency effect, Positions 37-48). In addition, two middle sections (Positions 13-24 and 25-36) were analyzed. A $2 \times 5 \times 2$ between-subjects analysis was performed on four separate positions within the test. The JOR scores for the beginning (Positions 1-12) were analyzed: no significant main effects were found for instruction, rehearsal group, or test order, and no first-order interactions were found for instruction rehearsal group, instruction $\times$ test order, or rehearsal group $\times$ test order. However, the second-order interaction of instruction $X$ rehearsal groups $\times$ test order was significant $[F(4,140)$ $=3.31, p<.05]$. A Newman-Keuls post hoc analysis did not reveal any mean differences, but the means indicate the the BWR subjects uninformed about the temporal order performed better when administered Test Order 2.

The analysis of Positions 13-24 did not turn up any significant effects. The JOR scores for Positions 25-36 and Positions 37-48 had results identical with those for Positions 13-24. These results indicate that the subjects responded to the treatment conditions consistently across all four separate positional blocks within the test list.

\section{Tape Recordings}

The FR, DR, FWR, and BWR rehearsal groups were required to rehearse into a microphone, and the subjects in the DR, FWR, and BWR groups followed instructions. All but a few subjects in the FR group had difficulty in rehearsing and simply stopped rehearsing the words audibly. Under these conditions it is difficult to differentiate between the FR and SR groups. It appears that forcing subjects to rehearse test pairs while imagining that they appeared on the blank lines was too difficult.

\section{DISCUSSION}

Disrupting the normal rehearsal pattern as one studies a serial list can lessen one's knowledge of serial order as shown in a serial position test, although this same finding was not observed in the JOR test. If one considers only the serial position data, there is clear support for the studyphase retrieval theory of Tzeng et al. (1979) and Tzeng and Cotton (1980). This model unequivocally states that position information is 
gradually acquired and strengthened as the subject rehearses in a stepwise, forward-moving pattern. Although the rehearsal strategy is certainly peculiar to the given subject and is thought to be disjointed and lacking in symmetry, it cannot, according to Tzeng, stand the strain of a backwards format and still convey the necessary position information. The subjects in the backward group were inferior in their knowledge of position, which is consistent with the study-phase retrieval model.

One can only speculate about the failure to find differences among groups with JOR scores. Tzeng (personal communication, April 1985) has stated that two distinct retrieval processes may be involved for serial position information and JOR responses. It may be that serial position scores are more sensitive to rehearsal strategy than JOR scores are.

With respect to the issue of automaticity, the present results can be interpreted as generally supportive of automaticity. Both serial position and JOR tests that bear directly on the encoding of temporal information show no overall differences between informed and uniformed instructional groups. This finding is contrary to the results reported by Michon and Jackson (1984), who found that an informed group was superior to an uninformed group on a temporal memory task. However, there was an operational difference between their uninformed group and ours. The uninformed subjects in our investigation were instructed to prepare for a subsequent free recall test, whereas Michon and Jackson instructed subjects to prepare for a recognition test. Michon and Jackson argue that free recall demands the reconstruction of the context, which includes temporal cues, in order to remember list words. It may be that our uninformed subjects were actively employing memory strategies that involved, among other things, a temporal component, because they anticipated a free recall test.

Michon and Jackson also maintain that temporal information will not facilitate a recognition test, because the items typically are presented individually (as, e.g., in a signal detection study), and because only the matching of intrinsic cues is needed; whereas temporal cues are needed for making judgments among words. One might conclude that they used a purer uninformed group than we did. Unfortunately, their position, for all its subtlety, is not supported by Toglia and Kimble (1976), who instructed informed subjects that a recognition test would follow and then failed to find differences between subjects who were informed and subjects who were uninformed.

Manipulation of the instructional variable has been described in the frequency-of-occurrence literature (Greene, 1984; Naveh-Benjamin \& Jonides, 1986; Sanders et al., 1987; Williams \& Durso, 1986). Sanders et al. (Experiment 2, 1987) did not find differences between instructional groups when the uninformed group was given nonspecific or general memory instructions for a subsequent-event frequency-ofoccurrence test. However, with a similar task, Greene (1984) did find significant differences when the uninformed group was not expecting any memory test. Matters are further complicated by research that indicates that when one uses incidental instructions of a nonspecific type, differences may or may not be found, depending upon the amount of time subjects have to encode the words (Naveh-Benjamin \& Jonides, 1986). Further manipulation of the instruction variable, within the temporal order literature, should help to determine the degree to which temporal coding is an automatic or effortful process.

\section{REFERENCES}

BLOCK, R. A. (1982). Temporal judgments and contextual change. Journal of Experimental Psychology: Learning, Memory, \& Cognition, 8, 530-544.

Fraisse, P. (1984). Perception and estimation of time. Annual Review of Psychology, 35, 1-36.

GreEnE, R. L. (1984). Incidental learning of event frequency. Memory \& Cognition, 12, 90-95.
Hasher, L., ZAcks, R. T. (1979). Automatic and effortful processes in memory. Journal of Experimental Psychology: General, 108, 356-388.

HiNRICHS, J. V. (1970). A two-process memory-strength theory for judgment of recency. Psychological Review, 77, 223-233.

Hintzman, D. L., Block, R. A., \&ummers, J. J. (1973). Contextual associations and memory for serial position. Joumal of Experimental Psychology, 97, 220-229.

KINTSCH, W. (1967). Memory and decision aspects of recognition learning. Psychological Review, 74, 496-504.

Michon, J. A., JACKSON, J. L. (1984). Attentional effort and cognitive strategies in the processing of temporal information. Annals of the New York Academy of Sciences, 423, 298-321.

Murdock, B. B., JR. (1974). Human memory: Theory and data. Potomac, MD: Erlbaum.

NAveh-Benjamin, M. (1987). Coding of spatial location information: An automatic process? Journal of Experimental Psychology: Learning, Memory, \& Cognition, 13, 595-605.

NAVEh-Benjamin, M., Jonides, J. (1986). On the automaticity of frequency coding: Effects of competing task load, encoding strategy, and intention. Journal of Experimental Psychology: Learning, Memory, \& Cognition, 12, 378-386.

Paivio, A., Yuille, J. C., \& Madigan, S. A. (1968). Concreteness, imagery, and meaningfulness values for 925 nouns. Journal of Experimental Psychology, 76, 1-25.

Palermo, D. S., Jenkins, J. J. (1964). Word association norms. Minneapolis: University of Minnesota Press.

Sanders, R. E., Gonzalez, E. G., MurPhy, M. D., Liddle, C. L., \& VitinA, J. R. (1987). Frequency of occurrence and the criteria for automatic processing. Journal of Experimental Psychology: Learning, Memory, \& Cognition, 13, 241-250.

SChNeIDER, W., \& SHIFfrin, R. M. (1977). Controlled and automatic human information processing: I. Detection, search, and attention. Psychological Review, 84, 1-66.

Shiffrin, R. M., \& SCHNeIDER, W. (1977). Controlled and automatic human information processing: II. Perceptual learning, automatic attending, and a general theory. Psychological Review, 84, 127-190.

THORNDIKE, E. L., \& LORGE, I. (1944). The teacher's word book of 30,000 words. New York: Columbia University Press.

TOGLIA, M. P., \& KIMBLe, G. A. (1976). Recall and use of serial position information. Journal of Experimental Psychology: Human Learning \& Memory, 2, 431-445.

Tulving, E., Madigan, S. A. (1970). Memory and verbal learning. Annual Review of Psychology, 21, 437-484.

TzEnG, O. J. L. (1976). A precedence effect in the processing of verbal information. American Journal of Psychology, 89, 577-599.

Tzeng, O. J. L., \& CotTon, B. (1980). A study-phase retrieval model of temporal coding. Journal of Experimental Psychology: Human Learning \& Memory, 6, 705-716.

Tzeng, O. J. L., LeE, A. T., \& Wetzel, C. D. (1979). Temporal coding in verbal information processing. Journal of Experimental Psychology: Human Learning \& Memory, 5, 52-64.

Willams, K. W., \& DuRso, F. T. (1986). Judging category frequency: Automaticity or availability? Journal of Experimental Psychology: Learning, Memory, \& Cognition, 12, 387-396.

YNTEMA, D. B., \& Trask, F. P. (1963). Recall as a search process. Journal of Verbal Learning \& Verbal Behavior, 2, 65-74.

Zimmerman, J., \& UNDERWOOD, B. J. (1968). Ordinal position knowledge within and across lists as a function of instructions in freerecall learning. The Journal of General Psychology, 79, 301-307.

(Manuscript received April 18, 1988.) 